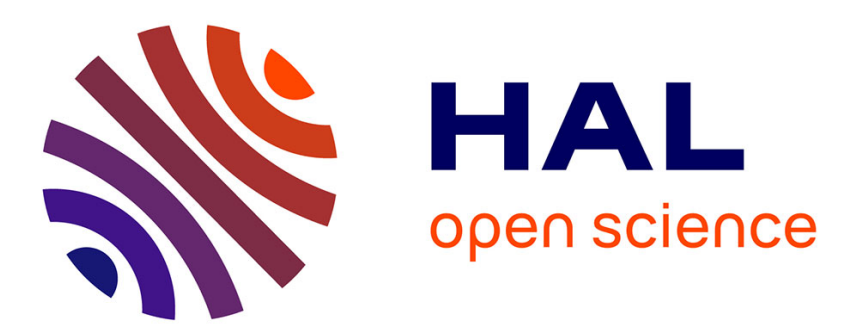

\title{
A Geometrical Wavelet Framework for the Time-Series Analysis of Full-Polarimetric Features
}

Davide Pirrone, Abdourrahmane Mahamane Atto, Emmanuel Trouve

\section{To cite this version:}

Davide Pirrone, Abdourrahmane Mahamane Atto, Emmanuel Trouve. A Geometrical Wavelet Framework for the Time-Series Analysis of Full-Polarimetric Features. 2020 IEEE Radar Conference (RadarConf20), Sep 2020, Florence, Italy. pp.1-6, 10.1109/RadarConf2043947.2020.9266403 . hal-03047702

\section{HAL Id: hal-03047702 \\ https://hal.science/hal-03047702}

Submitted on 8 Dec 2020

HAL is a multi-disciplinary open access archive for the deposit and dissemination of scientific research documents, whether they are published or not. The documents may come from teaching and research institutions in France or abroad, or from public or private research centers.
L'archive ouverte pluridisciplinaire HAL, est destinée au dépôt et à la diffusion de documents scientifiques de niveau recherche, publiés ou non, émanant des établissements d'enseignement et de recherche français ou étrangers, des laboratoires publics ou privés. 


\title{
A Geometrical Wavelet Framework for the Time-Series Analysis of Full-Polarimetric Features
}

\author{
Davide Pirrone, Abdourrahmane M. Atto and Emmanuel Trouvé University Savoie Mont Blanc, LISTIC, 74000 \\ Annecy, France. \\ e-mails: davide.pirrone@alumni.unitn.it; abdourrahmane.atto@univ-smb.fr; emmanuel.trouve@univ-smb.fr
}

\begin{abstract}
Polarimetric SAR (PolSAR) image time series have been employed for the analysis of temporal patterns of natural features in terms of the extended polarimetric scattering properties. However, the time series provide a rich scattering information that can be used for tracking and analyzing the evolution of targets, individuating smooth and/or abrupt changes. In this work we propose a wavelet framework that exploits the information from polarimetric features and analyze them to both mitigate the speckle effect on the multi-temporal information and improve the targets homogeneity using the multi-temporal information. The framework combines the powerful description from the main polarimetric decomposition features and the temporal analysis using geometrical wavelet transform. The analysis is applied on a multi-temporal polarimetric dataset of Radarsat- 2 images acquired over the Argentière glacier site.
\end{abstract}

\section{Index Terms}

Polarimetric SAR, Time series, Incoherent Polarimetric Decomposition, Wavelet Temporal Operator, Multi-temporal Analysis, Geometrical wavelet

\section{INTRODUCTION}

In the last decade, large datasets of multi-temporal SAR and Polarimetric SAR (PolSAR) images have been delivered to the scientific community. This led to a large interest in the use of multi-temporal data for robust classification [1], for change detection (CD) [2] and for the multi-temporal analysis of natural features (e.g., snow monitoring [3], soil moisture analysis [4], glacier displacement [5]). A large part of the SAR literature tackled the CD considering a bi-temporal problem, with different approaches [6]-[9]. A smaller part has been devoted to the analysis of PolSAR image time series for the discrimination of both abrupt and gradual changes [10].

Wavelet transform is a very important tool of analysis for tracing and individuating the characteristics of the signal in terms of low and high frequencies. In SAR image analysis, the frequencies have been typically considered in the spatial domain (i.e., multi-scale CD analysis [11], SAR texture analysis [12]). A few of works considered the application of wavelets in the temporal domain, which can characterize changes with different temporal behavior on a image time series [5]. However, the literature has mainly considered the application of the standard wavelet to features characterized by statistics on both coherent and incoherent imaging principles (i.e., additive and multiplicative noise for optical and PolSAR intensity data, respectively). These differences may affect the performance of the wavelet decomposition. Thus, an alternative wavelet formulation (i.e., geometrical wavelet) under the assumption of multiplicative noise has been derived in [5], but it has not been exploited yet in the PolSAR image analysis. This wavelet framework represents a powerful tool for characterizing the variability of polarimetric features indicating power information [13].

In this work, we propose the use of the geometrical wavelet framework for the multi-temporal analysis of polarimetric features from full-polarimetric SAR image time series. In particular, the features result from the Yamaguchi polarimetric decomposition [13], which maps the different scattering mechanisms. The analysis considers the application of the wavelet framework along the temporal domain, in order to characterize the pixel-based temporal evolution of the target in terms of the polarimetric features. The framework aims at both: i) mitigating the speckle effect on the multi-temporal change information, which can be later used for change detection applications; and ii) characterizing the temporal evolution of the change along the time series. Experimental results on a multi-temporal PolSAR dataset, acquired from Radarsat-2 on the Argentière glacier area (France), show the effectiveness of the proposed framework.

The paper is structured as follows: in Section II we describe the polarimetric features and the wavelet strategies considered for the analysis. Section III illustrates the experimental results on a multi-temporal PolSAR dataset. Finally, in Section IV we trace the conclusions and the future developments of this work. 


\section{Methodology}

Let us consider a time-series of $N$ PolSAR images $I_{t}, t=1, \ldots, N$. As the natural targets present incoherent scattering, polarimetric information of each image is represented with second-order scattering information of Coherency matrix $T$.

$$
T=\frac{1}{L} \sum_{L} k_{P} k_{P}^{H}
$$

being $k=\left[S_{H H}+S_{V V}, S_{H H}-S_{V V}, 2 S_{H V}\right] / \sqrt{2}$ the complex scattering vector in the Pauli basis and $L$ the number of looks. $T$ matrix is an hermitian matrix with a sparse information content. Incoherent polarimetric decompositions provide a representation of the physical information of $T$ in terms of few features. The literature provides two large decomposition classes: model-based and eigenvector-based decompositions. Among the model-based ones, Yamaguchi proposed a composition of contributions of surface $(s)$, double bounce $(d)$, volume $(v)$ and helix scattering $(h)$.

$$
T=\sum_{n} f_{n} T_{n}
$$

being $f_{n}, T_{n}$ the power coefficient and the coherency matrix associated to the scattering mechanism $n, n \in s, d, v, h$, respectively. $T_{n}$ is modeled based on the polarimetric properties assumed for the target. From here, power associated to the different mechanisms can be derived as $P_{d}=f_{d}\left(1+|\alpha|^{2}\right), P_{s}=f_{s}\left(1+|\beta|^{2}\right), P_{v}=f_{v}$ and $P_{h}=f_{h}$, being $\alpha, \beta$ complex model parameters. The power sum corresponds to the span of the matrix $T$ [13].

Let $X$ be one of the polarimetric features above illustrated, $X \in\left\{P_{s}, P_{v}, P_{d}, P_{h}\right\}$. In the literature, wavelet-based analysis has proved to be an effective tool for pattern analysis, being applied on spatial and/or temporal domain. In this work, we focus the analysis on the temporal direction only, with a corresponding pixel-based spatial analysis. Thus, for each set of image spatial coordinates $(r, c)$ of the feature $X$, a multi-temporal vector $X_{t}=X_{t}(r, c), t=1, \ldots, T$ is derived.

The application of the wavelet transform on $X_{t}$ yields two components, associated to approximation and detail. These are derived as convolution of the input signal with two filter responses $W_{A}, W_{D}$. Without loss of generality, the wavelet is assumed not considering the decimation step (i.e., Stationary Wavelet, SW). The application of the classical (i.e., arithmetical) wavelet transform, assuming the noise affecting the signal as additive, considers the classical convolution operation $\circledast$ between the input and the wavelet filter response $W_{b}(\cdot)$.

$$
X_{t}^{\left(W_{a b}\right)}=\sum_{l=0}^{L-1} W_{b}(l) X_{t-l}=W_{b} \circledast X_{t}
$$

being $X_{t}^{\left(W_{a b}\right)}$ the temporal sequence of the wavelet component $b$ for the feature $X$, computed with arithmetical wavelet and $b$ an indicator for the approximation (i.e., $b=A$ ) and detail component (i.e., $b=D$ ), respectively. On the other hand, a geometrical convolution operation () is considered for geometrical wavelets [5], in order to better take into account the presence of multiplicative noise on the signal.

$$
\begin{aligned}
X_{t}^{\left(W_{g b}\right)} & =\exp \left[\sum_{l=0}^{L-1} W_{b}(l) \log \left(X_{t-l}\right)\right] \\
& =\prod_{l=0}^{L-1}\left(X_{t-l}\right)^{W_{b}(l)}=W_{b} \odot X_{t}
\end{aligned}
$$

being $X_{t}^{\left(W_{g b}\right)}$ the temporal sequence of the wavelet component $b$ for the feature $I$, computed with geometrical wavelet.

In this work, a single scale level for the wavelet analysis has been considered. However, a multi-scale analysis can be considered for signals with large length, by defining the $X$ as the approximation component at scale 0 and applying the wavelet filtering to the approximation component at each scale level. For a set of $K+1$ scales, this provides an approximation component (computed at scale $K$ ) and a set of $K$ detail components.

Several wavelet families have been defined in the literature for signal processing (e.g. Symlet, Daubechies). Among them, the Haar-1D has a large importance, because of the easiness of representation for its filter responses, with coefficients $W_{A}=[1,1] / C_{0}$ and $W_{D}=[1,-1] / C_{0}$ for the approximation and detail component respectively, where the normalization constant $C_{0}$ is either 2 or $\sqrt{2}$. When applied to a temporal series, the Haar approximation output represents the temporal geometrical mean of the two subsequent images. This is an information that can be exploited for filtering of unchanged areas [14] (see eq. 6). On the other hand, the detail output can be associated to the multi-temporal comparison of values at consequent time instants. In particular, for Haar-1D, the output represents the pixel-based image ratio, widely used for the multi-temporal SAR image analysis [2] (see eq. 7).

$$
X_{t}^{\left(W_{g}\right)}=\left\{X_{t}^{\left(W_{g A}\right)}, X_{t}^{\left(W_{g D}\right)}\right\}
$$




$$
\begin{aligned}
& X_{t}^{\left(W_{g A}\right)}=W_{A} \odot X_{t}=\exp \left[\frac{\log \left(X_{t-1}\right)+\log \left(X_{t}\right)}{C_{0}}\right] \\
& X_{t}^{\left(W_{g D}\right)}=W_{D} \odot X_{t}=\exp \left[\frac{\log \left(X_{t-1}\right)-\log \left(X_{t}\right)}{C_{0}}\right]
\end{aligned}
$$

\section{EXPERIMENTAL RESULTS}

In order to prove the effectiveness of the proposed information representation for PolSAR image time series, a multi-temporal dataset of full-pol images with size $1024 \times 1024$ was considered. The dataset has been acquired by Radarsat- 2 mission in the area of the Argentière glacier (France) in the period between January 29 and June 29, 2009. The area is characterized by changes associated to glacier evolution. The dataset includes 7 full-polarimetric SAR images, each with a geometrical resolution of 8 meters and incidence angle of $32^{\circ}$ (see Fig. 1a-1b). A preliminary processing of the image set has been conducted in terms of radiometric calibration, generation of the coherency matrix $T$ and polarimetric image despeckling (i.e., Refined Lee filter $7 \times 7$ ) and co-registration, respectively. For this dataset, 6 regions of interest have been selected for a local quantitative assessment based on prior knowledge of the scene, defined as $\{R 1, \ldots, R 6\}$.

For each image, polarimetric features have been derived with the power-based Yamaguchi decomposition on a local window

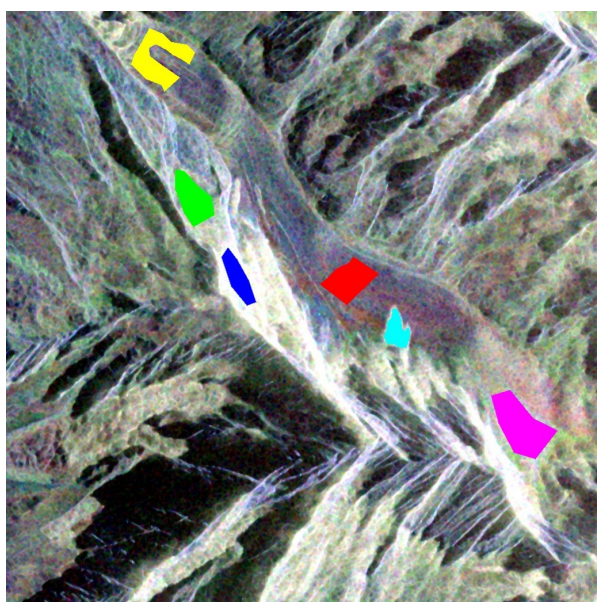

(a)

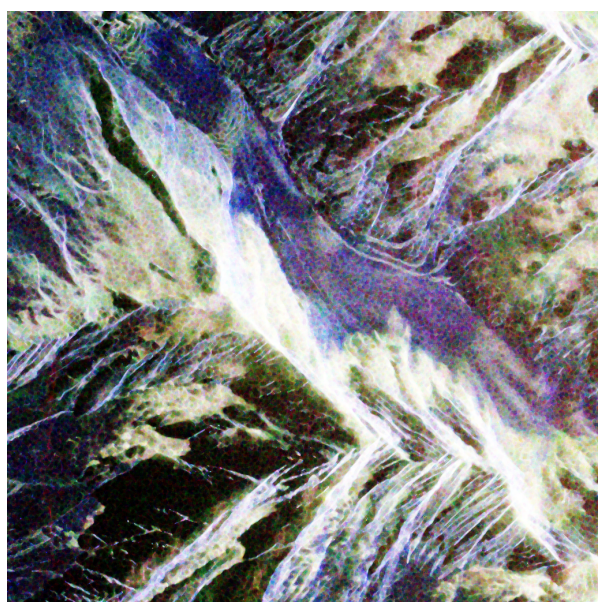

(b)

Fig. 1: Pauli false color composition for times: $t_{1}$ (a); $t_{5}$, (b). Corresponding dates: January 29, May 05, 2009 with 6 regions of interest. HH + VV: Blue; HH - VV: Red; HV: Green.

using ESA Sentinel Toolbox software. The window size has been set to $5 \times 5$ as a tradeoff between scene homogeneity and preservation of local edges. For each of the Yamaguchi features, the framework has been applied on a pixel basis, selecting Haar-1D as wavelet family. Both geometrical (GW) and arithmetical (AW) wavelets have been tested on the dataset. Qualitative and quantitative analysis were then focused on the set of regions $\{R 1, . . R 6\}$, selected based on prior knowledge of the area.

For each feature, two parameters were derived on each region for a numerical assessment. The two parameters consider alternatively the two wavelet components and investigate the effectiveness of the wavelet approach in: i) enhancing the image contrast (i.e., approximation component) and ii) following the temporal evolution (i.e., detail component). Both parameters have been investigated by considering the Coefficient of Variation $(\mathrm{CoV})$ on the regions of interest for both the features and the corresponding wavelet components. For the feature $Y$, the $\mathrm{CoV}$ is defined as follows.

$$
\operatorname{CoV}(Y)=\frac{\sqrt{E\left\{(Y-E\{Y\})^{2}\right\}}}{E\{Y\}}
$$

being the expectation $E\{\cdot\}$ evaluated as ensemble averaging and $Y$ either the polarimetric feature $X$ or one of its wavelet components.

\section{A. Approximation Component}

As the approximation component conveys information about the temporal mean, it represents a smoothed version of the temporal trend for the considered feature. Fig. $2 \mathrm{a}-2 \mathrm{~d}$ illustrate the $\mathrm{GW}$ approximation component of time pair $t=t_{4}, t_{5}$ for the four Yamaguchi polarimetric features. The approximation feature well maps the average of the values of the two single-time images (see Pauli components in Fig. 1a-1b), mitigating the presence of possible outliers in homogeneous regions. A comparison between the approximation components from GW and AW has been conducted. Fig. 3a-3d illustrate the histograms of the approximation components obtained with the two wavelets on the Yamaguchi polarimetric features, reported in logarithmic 
scale. The GW approximation operator yields histograms with larger value interval (e.g., about $45 \mathrm{~dB}$ and $30 \mathrm{~dB}$ for volume component with GW and AW, respectively), thus enhancing the image dynamics and favoring image analysis. Smaller mean value is also observed for the GW, compared to the AW.

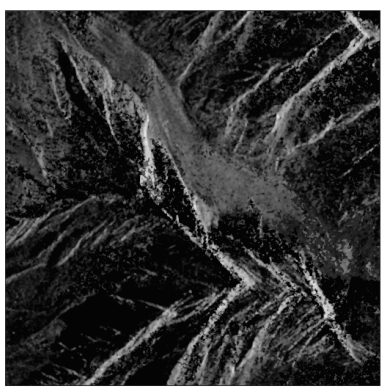

(a)

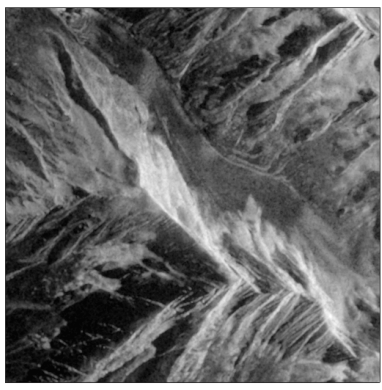

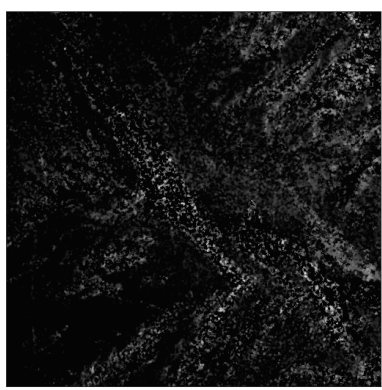

(b)

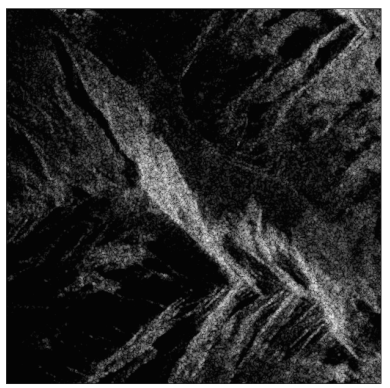

(d)

Fig. 2: GW approximation for surface (a), double bounce (b), volume (c) and helix components (d). Features are evaluated for time pair $t_{4}, t_{5}$.

A local analysis has been performed on the six regions of interest. Fig. 4 shows as example the temporal trend of $C o V$ values for one of the region (i.e., $R 4$ ). From the plots, the approximation geometrical wavelet (red lines) shows $C o V$ values globally higher than those for the arithmetic wavelet (blue lines), corresponding to a smaller noise reduction. Looking more in detail, these values are associated to the reduction of both mean and standard deviation. This effect might be in part explained by the interaction between the spatial and temporal analysis processes in the two cases, together with the sensitivity of the framework to the change events present in the time-series and more or less gradually affecting the target [14]. This is associated to the reduction of the image standard deviation and thus an enhancement of the image contrast, when using the geometric wavelet instead of the arithmetical one. Globally, the geometrical wavelet seems to typically better follow and enhance the evolution trend of the polarimetric features compared to those from arithmetical wavelet.

For the numerical assessment of filtering performance, we propose a parameter $\Delta$ called Averaged Difference Variation Index (ADVI) and defined by:

$$
\Delta=\overline{\operatorname{CoV}\left(X_{t}^{W A}\right)}-\overline{C o V\left(X_{t}\right)}
$$

where $X_{t}$ and $X_{t}^{S W A}$ denote respectively the input time series and their wavelet approximation computed with either the $\mathrm{AW}$ or $\mathrm{GW}$, and $\overline{(\cdot)}$ represents the average value computed, for each region, along the temporal domain. Table I shows the ADVI values obtained for the different regions and features, with arithmetical and geometrical approach, respectively. This table highlights that GW approach, in general, shows more variability than AW approach, with GW values ranging from 1.305 to 5.861, while AW ones from -0.8451 to 0.574 for double-bounce component on the regions of interest. This can be due to the fact that input time series is associated with a large amount of radiometric changes over the whole test site. Similar considerations can be traced for the other polarimetric components.

\section{B. Detail Component}

The second analysis focused on the wavelet detail component, which provides information about the temporal variation of the corresponding feature along the temporal axis. Fig. 5a-5d shows the GW detail component of time pair $t_{4}, t_{5}$, for the four Yamaguchi features. The detail component highlights well the presence of positive and negative changes in the image pair, mitigating the effect of speckle noise in the change detection, as other SAR multi-temporal comparison operators. A comparison of the histograms for GW and AW detail components for the features is illustrated in Fig. 7a - 7d in logarithmic 


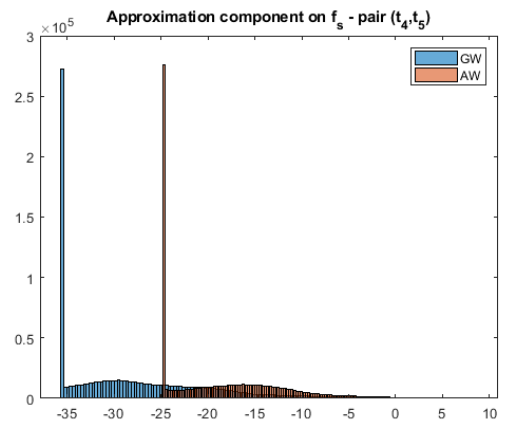

(a)

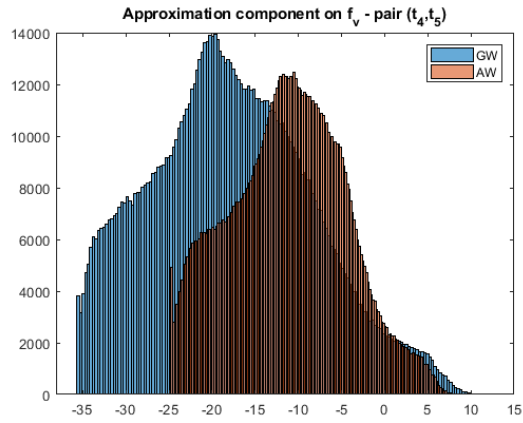

(c)

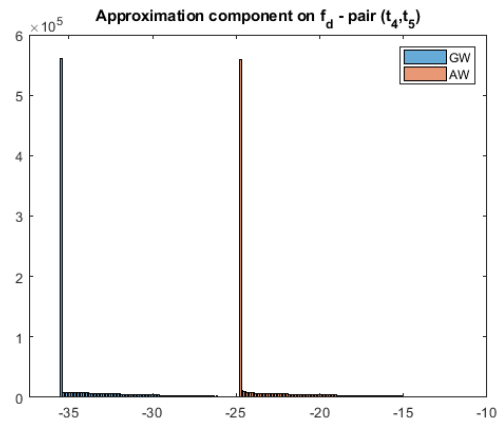

(b)

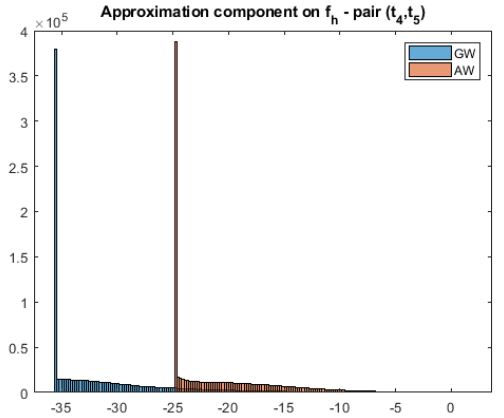

(d)

Fig. 3: Histogram comparison of GW (light blue) and AW (orange) approximation components, reported in log scale, for the four polarimetric features (pair $\left.t_{4}, t_{5}\right)$ : surface (a); double bounce (b); volume (c) and helix (d).

TABLE I: ADVI $\Delta$ values for the different features on the local regions $R 1-R 6$.

\begin{tabular}{|c|c|c|c|c|c|c|}
\hline$\Delta$ & $\mathbf{R 1}$ & $\mathbf{R 2}$ & $\mathbf{R 3}$ & $\mathbf{R 4}$ & $\mathbf{R 5}$ & $\mathbf{R 6}$ \\
\hline$P_{d}$ AW & -0.636 & -0.438 & 0.574 & -0.851 & -0.139 & -0.085 \\
\hline$P_{d} \mathbf{G W}$ & 5.620 & 3.838 & 5.154 & 1.305 & 3.324 & 5.861 \\
\hline$P_{h} \mathbf{A W}$ & -0.512 & -0.280 & -0.944 & -0.221 & -0.659 & -0.130 \\
\hline$P_{h} \mathbf{G W}$ & -0.690 & 2.247 & 0.430 & 1.577 & 0.883 & 3.088 \\
\hline$P_{s} \mathbf{A W}$ & -0.298 & -1.223 & 0.430 & 0.241 & -1.083 & 0.150 \\
\hline$P_{s} \mathbf{G W}$ & 0.373 & 6.8362 & 1.912 & 1.785 & 4.688 & 8.959 \\
\hline$P_{v} \mathbf{A W}$ & -0.363 & -1.070 & -0.240 & -0.211 & -0.638 & -0.435 \\
\hline$P_{v} \mathbf{G W}$ & 0.307 & 1.391 & 0.269 & 2.212 & 0.363 & 0.724 \\
\hline
\end{tabular}

scale. Because of the definition of the AW detail operator, negative values might be present and logarithmic representation would not be valid. Therefore, the histogram qualitative comparison considered the translation of the AW detail minimum to zero. AW histograms are characterized by a smaller range, with a non-zero mean in the general case. These aspects confirm a larger presence of residual speckle in the AW component, compared to that for GW, because of the different representation of the multi-temporal information. This aspect impacts on the statistical properties and in the change detection performances.

The analysis focused on the set $R 1, \ldots, R 6$. Fig. 6 illustrates the temporal trend of the wavelet detail components together with that of the corresponding Yamaguchi features on the region $R 4$. The plot highlights how $\mathrm{GW}$ is able to better mitigate the speckle effect and follow the temporal evolution of the feature, while, for AW, the change of the area is wounded by local residual speckle effects (e.g., see blue curves in Fig. 6). This can be justified by the fact that, under the Wishart assumption for matrix $T$, a Gamma distribution can be considered for modeling the different power terms [15].

Numerical assessment has been conducted on six regions by considering a measure of the total temporal gradient. A coefficient $T T G$ has been defined for the feature $X$ as:

$$
T T G=\left\{\begin{array}{cc}
\sum_{t}\left|\nabla_{t} \operatorname{CoV}\left(X_{t}\right)\right| & \text { for } X_{t} \\
\sum_{t} \operatorname{CoV}\left(X_{t}^{(W a D)}\right) & \text { for } X_{t}^{(S W a D)} \\
\sum_{t} \operatorname{CoV}\left(X_{t}^{(W g D)}\right) & \text { for } X_{t}^{(S W g D)}
\end{array}\right.
$$

Table II shows the values obtained for the different regions on both the features and wavelet detail components. The geometrical wavelet presents values much closer to those of the polarimetric features, compared to those of the arithmetical wavelet. This confirms the effectiveness of the geometrical wavelet framework in mitigating speckle effect on multi-temporal information from Yamaguchi polarimetric features. 

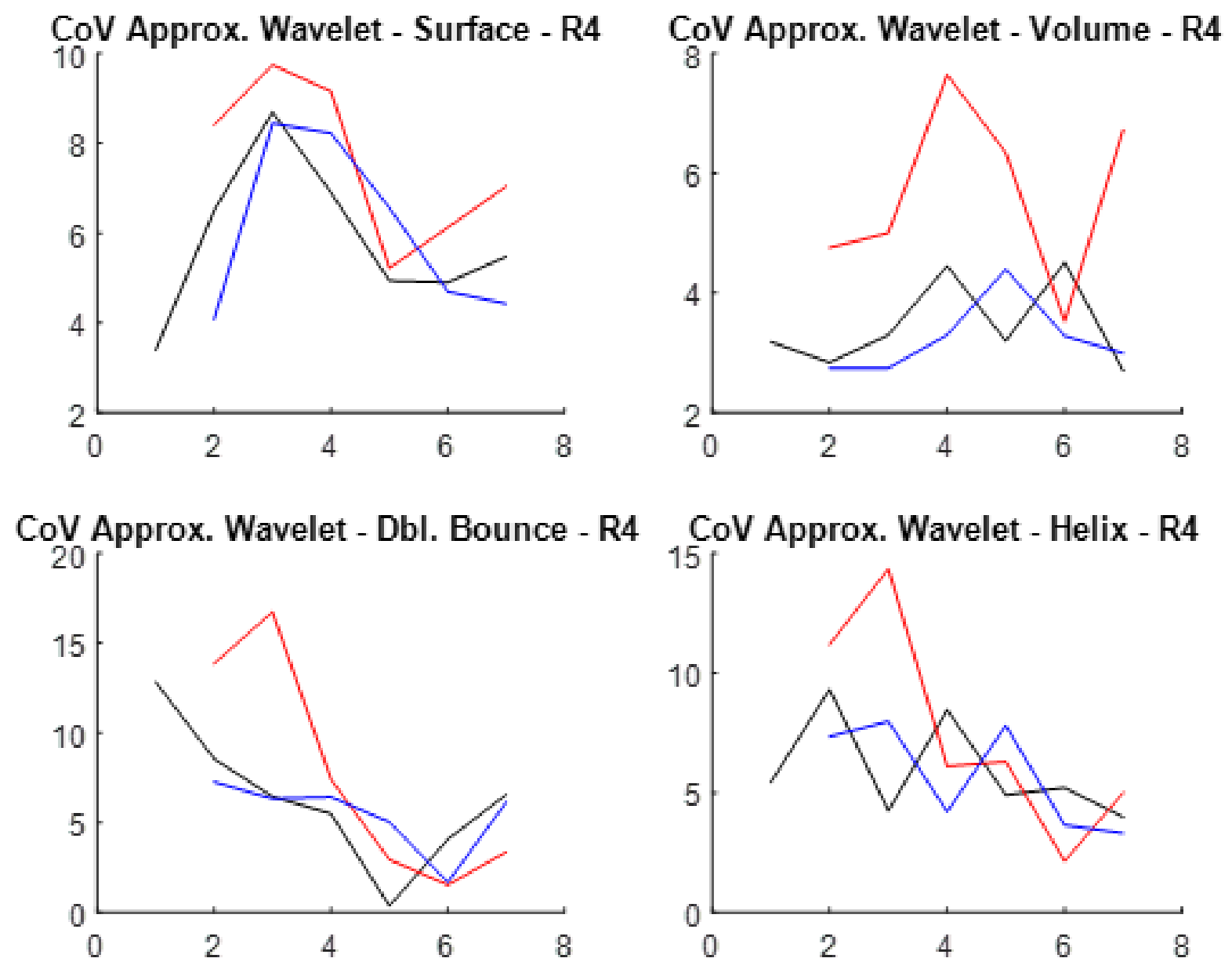

Fig. 4: Temporal trend for polarimetric features and wavelet Approximation (Region R4); Polarimetric Feature (black); Arithmetical Wavelet (blue); Geometrical Wavelet (red).

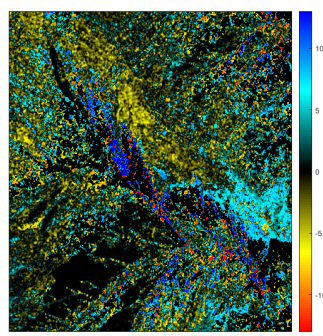

(a)

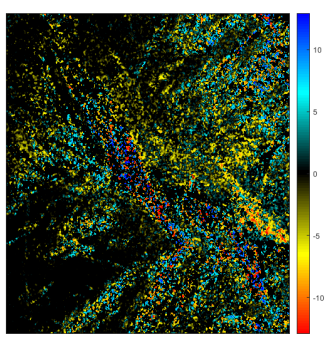

(b)

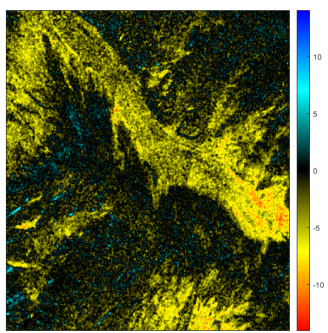

(c)

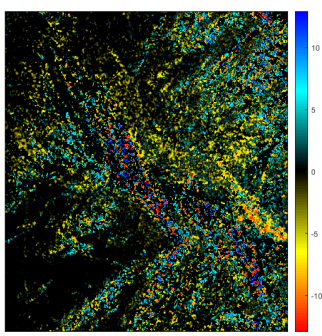

(d)

Fig. 5: GW detail for surface (a), double bounce (b), volume (c) and helix components (d) for time pair $t_{6}, t_{7}$.

\section{CONCLUSIONS}

In this work, we proposed the use of a framework based on geometrical wavelet transform for analyzing polarimetric features from Yamaguchi decomposition. The analysis considered the application of the geometrical wavelet along the temporal domain for separating the temporal average and the variation information on a polarimetric image time series. Experimental analysis has been conducted on a multi-temporal polarimetric dataset and focused on local regions of interest, selected based on the prior knowledge of the scene. The results showed the effectiveness of the temporal framework in tracking the temporal evolution of the targets and displaying the change information. These aspects might be an immediate benefit to be integrated into change detection methodologies. Future developments aim in investigating the use of the temporal wavelet analysis for combinations 

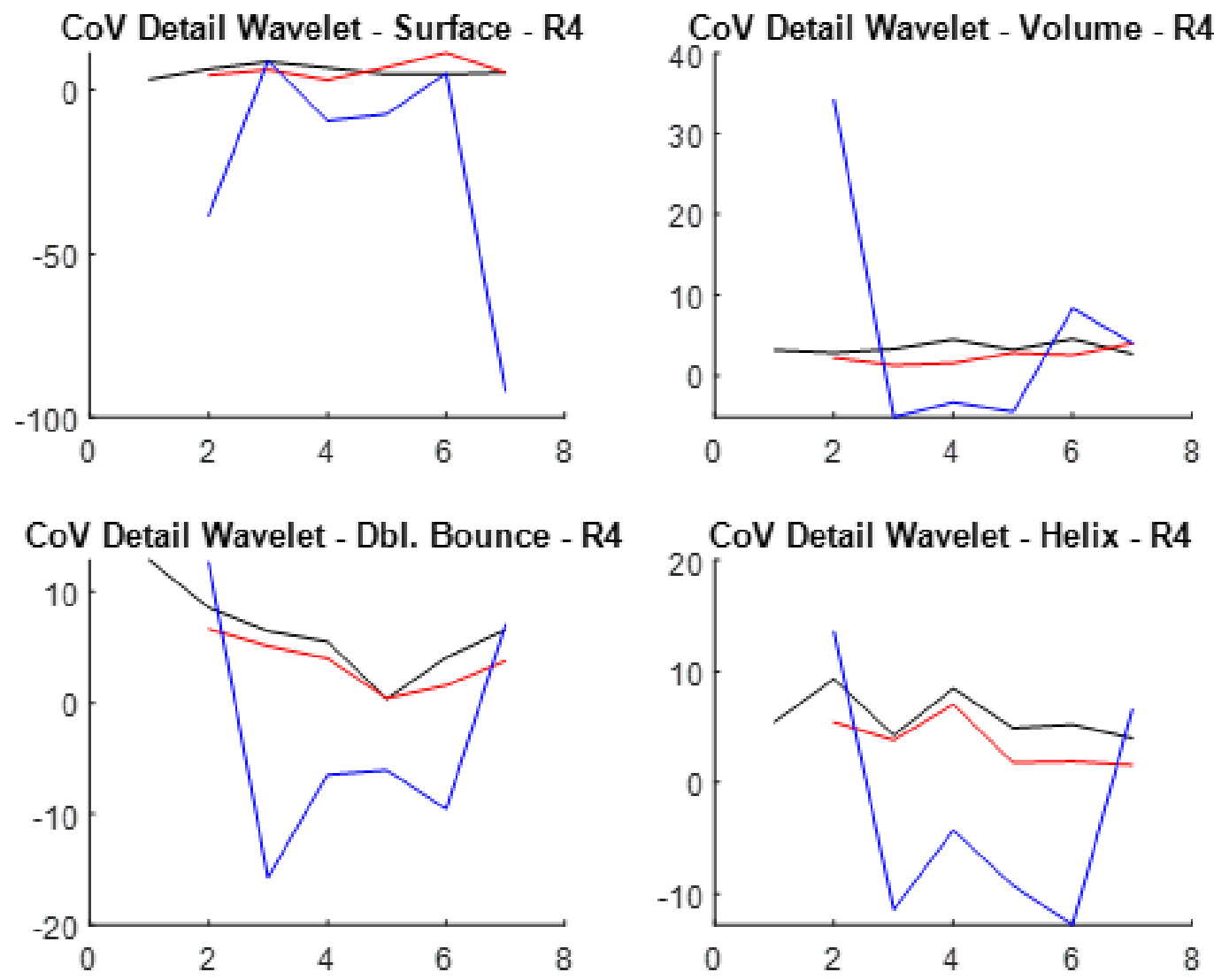

Fig. 6: Temporal trend for polarimetric features and wavelet Detail (Region $R 4$ ); Polarimetric Feature (black); Arithmetical Wavelet (blue); Geometrical Wavelet (red).

TABLE II: $T G G$ values for the different features on the local regions $R 1-R 6$.

\begin{tabular}{|c|c|c|c|c|c|c|}
\hline$T T G$ & R1 & R2 & R3 & R4 & R5 & R6 \\
\hline$P_{d}$ Feature & 11.22 & 34.168 & 24.57 & 18.67 & 19.39 & 30.69 \\
\hline$P_{d}$ AW & 54.39 & 137.303 & 143.20 & 53.53 & 50.22 & 95.61 \\
\hline$P_{d}$ GW & $\mathbf{1 9 . 7 1}$ & $\mathbf{4 7 . 1 6 8}$ & $\mathbf{3 8 . 2 2}$ & $\mathbf{2 1 . 5 3}$ & $\mathbf{3 4 . 0 5}$ & $\mathbf{4 4 . 8 8}$ \\
\hline$P_{h}$ Feature & 10.34 & 19.490 & 7.21 & 18.25 & 14.16 & 11.69 \\
\hline$P_{h}$ AW & 52.07 & 116.372 & 76.54 & 58.95 & 57.23 & 106.39 \\
\hline$P_{h}$ GW & $\mathbf{1 2 . 8 4}$ & $\mathbf{3 7 . 1 7 8}$ & $\mathbf{6 2 . 4 8}$ & $\mathbf{2 1 . 5 7}$ & $\mathbf{3 6 . 5 6}$ & $\mathbf{4 1 . 6 4}$ \\
\hline$P_{s}$ Feature & 1.16 & 11.997 & 17.68 & 9.67 & 9.94 & 37.72 \\
\hline$P_{s}$ AW & 26.14 & 44.301 & 60.91 & 101.07 & 62.91 & 118.61 \\
\hline$P_{s}$ GW & $\mathbf{3 3 . 8 2}$ & $\mathbf{3 1 . 9 1}$ & $\mathbf{3 0 . 1 0}$ & $\mathbf{3 8 . 2 0}$ & $\mathbf{3 5 . 6 0}$ & $\mathbf{5 5 . 6 8}$ \\
\hline$P_{v}$ Feature & 3.45 & 14.33 & 2.06 & 6.34 & 9.01 & 3.75 \\
\hline$P_{v}$ AW & 443.88 & 36.31 & 33.54 & 59.51 & 43.92 & 44.69 \\
\hline$P_{v}$ GW & $\mathbf{1 7 . 5 2}$ & $\mathbf{1 0 . 0 5}$ & $\mathbf{9 . 4 9}$ & $\mathbf{1 4 . 2 3}$ & $\mathbf{1 2 . 7 3}$ & $\mathbf{1 3 . 4 6}$ \\
\hline
\end{tabular}

of multiple polarimetric features. Moreover, the use of different wavelet filters (e.g., Daubechies) and/or the use of a multi-scale paradigm applied in the temporal domain would be suitable for highlighting phenomena with different temporal evolution.

\section{REFERENCES}

[1] D. Bargiel, "A new method for crop classification combining time series of radar images and crop phenology information," Remote Sensing of Environment, vol. 198, pp. 369-383, 2017.

[2] F. Bovolo and L. Bruzzone, "The time variable in data fusion: A change detection perspective," IEEE Geosci. Remote Sens. Mag, vol. 3, no. 3, pp. 8-26, 2015.

[3] A. Muhuri, S. Manickam, and A. Bhattacharya, "Scattering mechanism based snow cover mapping using radarsat-2 c-band polarimetric sar data," IEEE Journal of Selected Topics in Applied Earth Observations and Remote Sensing, vol. 10, no. 7, pp. 3213-3224, 2017.

[4] S. Paloscia, S. Pettinato, E. Santi, C. Notarnicola, L. Pasolli, and A. Reppucci, "Soil moisture mapping using sentinel-1 images: Algorithm and preliminary validation," Remote Sensing of Environment, vol. 134, pp. 234-248, 2013.

[5] A. M. Atto, E. Trouvé, J.-M. Nicolas, and T. T. Lê, "Wavelet operators and multiplicative observation models-application to sar image time-series analysis," IEEE Transactions on Geoscience and Remote Sensing, vol. 54, no. 11, pp. 6606-6624, 2016. 


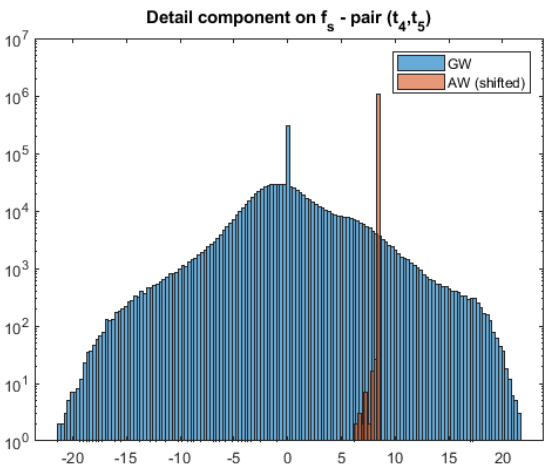

(a)

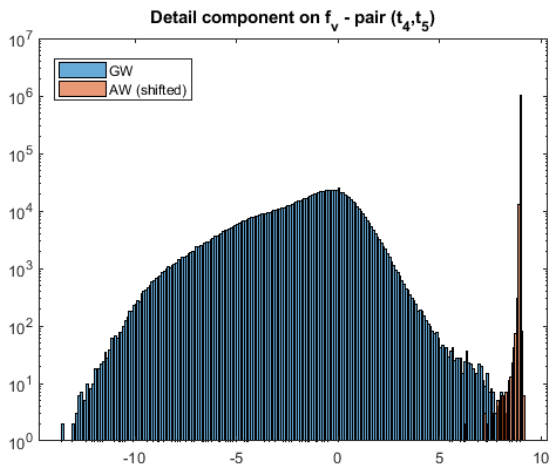

(c)

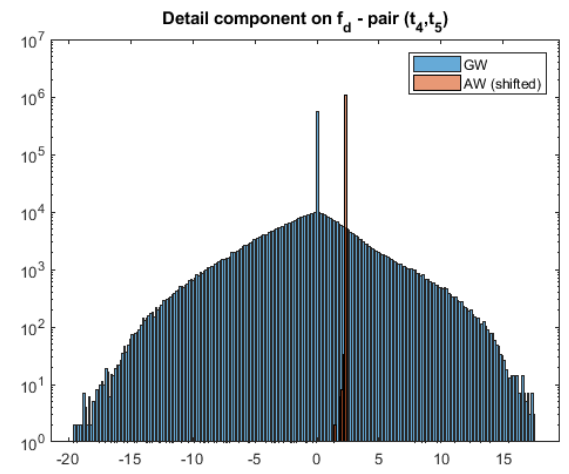

(b)

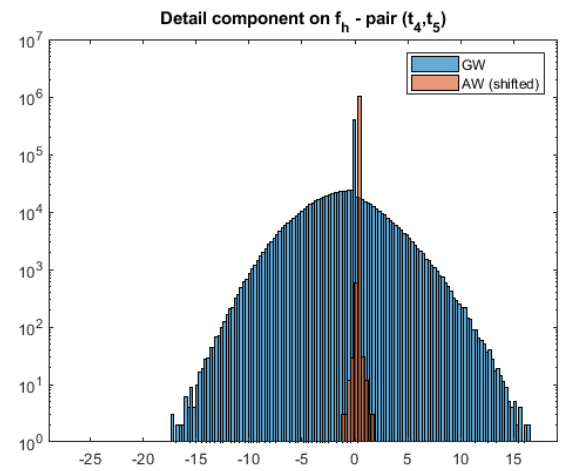

(d)

Fig. 7: Histogram comparison of GW (light blue) and AW (orange) detail components, reported in log scale, for the four polarimetric features (pair $t_{4}, t_{5}$ ): surface (a); double bounce (b); volume (c) and helix (d).

[6] K. Conradsen, A. A. Nielsen, J. Schou, and H. Skriver, "A test statistic in the complex wishart distribution and its application to change detection in polarimetric sar data," IEEE Transactions on Geoscience and Remote Sensing, vol. 41, no. 1, pp. 4-19, 2003.

[7] V. Akbari, S. N. Anfinsen, A. P. Doulgeris, T. Eltoft, G. Moser, and S. B. Serpico, "Polarimetric sar change detection with the complex hotelling-lawley trace statistic," IEEE Transactions on Geoscience and Remote Sensing, vol. 54, no. 7, pp. 3953-3966, 2016.

[8] A. Marino and I. Hajnsek, "A change detector based on an optimization with polarimetric sar imagery," IEEE Transactions on Geoscience and Remote Sensing, vol. 52, no. 8, pp. 4781-4798, 2014.

[9] S.-W. Chen and M. Sato, "Tsunami damage investigation of built-up areas using multitemporal spaceborne full polarimetric sar images," IEEE Transactions on Geoscience and Remote Sensing, vol. 51, no. 4, pp. 1985-1997, 2013.

[10] W. Liu, J. Yang, J. Zhao, H. Shi, and L. Yang, "An unsupervised change detection method using time-series of polsar images from radarsat-2 and gaofen-3," Sensors, vol. 18, no. 2, p. 559, 2018.

[11] J. Inglada and M. Grégoire, "A new statistical similarity measure for change detection in multitemporal sar images and its extension to multiscale change analysis," IEEE transactions on geoscience and remote sensing, vol. 45, no. 5, pp. 1432-1445, 2007.

[12] S. Fukuda and H. Hirosawa, "A wavelet-based texture feature set applied to classification of multifrequency polarimetric sar images," IEEE Transactions on Geoscience and Remote Sensing, vol. 37, no. 5, pp. 2282-2286, 1999.

[13] Y. Yamaguchi, T. Moriyama, M. Ishido, and H. Yamada, "Four-component scattering model for polarimetric sar image decomposition," IEEE Transactions on Geoscience and Remote Sensing, vol. 43, no. 8, pp. 1699-1706, 2005.

[14] G. Quin, B. Pinel-Puyssegur, J.-M. Nicolas, and P. Loreaux, "Mimosa: An automatic change detection method for sar time series," IEEE Transactions on Geoscience and Remote Sensing, vol. 52, no. 9, pp. 5349-5363, 2013.

[15] S. Dauphin, R. D. West, R. Riley, and K. M. Simonson, "Semi-supervised classification of terrain features in polarimetric sar images using h/a/ $\alpha$ and the general four-component scattering power decompositions," in 2014 48th Asilomar Conference on Signals, Systems and Computers, pp. 167-171, IEEE, 2014. 